\title{
Is There an Increase in Psychological Problems Among Chinese College Students During COVID-19
}

\author{
Xiaochen Liang ${ }^{*} \dagger$ \\ ${ }^{1}$ School of York University, Toronto, Ontario, Canada

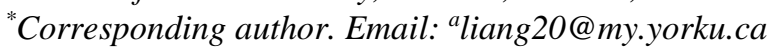

\begin{abstract}
The emergence of COVID-19 has been a serious public issue around the whole world and has caused huge change to people's daily life. Governments have taken series of actions in order to block the infections between individuals, such as lock down of public sites and self-quarantine. As the cases decreased, mental health problems appeared among Chinese college students. By reviewing existing articles, the present study aimed to find and summarize evidence that can investigate the mental health problem status during COVID-19. The results showed that there was an increase in psychological problems among Chinese college students, and there were also several ways to improve the abilities that can help the mental health stay on a healthy scale, including keeping physical activities, and asking professionals for help.
\end{abstract}

Keywords: COVID-19, mental health problems, Chinese college students, way to improve psychological problems

\section{INTRODUCTION}

The world has been changed by a newly discovered disease called COVID-19. The world health organization defined COVID-19 as a highly infectious disease and the virus could transform between humans. The main symptoms included difficulty breath and shortness of breath [1]. Tons of people have died because of COVID-19. The first case was found in Wuhan, Hubei, China and it soon spread around the world. Since the cases in China increased sharply, the Chinese government announced a lockdown policy which indicated that all people must stay at home. People started to work and study at home. The lockdown policy have caused an obvious result that the number of cases decreased within several months and remained stable from then on. However, with the decrease of the cases, mental health problems increased. The accumulation of negative effects of lockdown on individuals' psychological functions such as depression, sleeping problems, and anxiety gradually showed up. Mental health problem became a global issue after the pandemic developed. The present research will mainly focus on the mental health problems among Chinese college students during COVID-19.

During the special time period of COVID-19, students were required to stay at home and study online.
The challenges appeared during the lockdown. According to Chen et al. [2], the lockdown primarily raised two kinds of challenges for college students. First, the lack of socialization Students lost the freedom of having outdoor activities and the opportunities of socializing face-to-face with their friends and teachers as they could before lockdown, and this lack of social activities can cause mental health problems and even depression. Secondly, being disorganized in their life can also be a challenge for college students. Students have different life routines compared with the day in school. They have to change their daily routine to accommodate the new lifestyle. During the accommodation, students have several problems such as getting up late, spending more time on their devices, and so on. Hence, they need time to accept the abrupt change and overcome the uncomfortable feeling caused by the change. Different people have different environmental adaptive capabilities. For those who have the low environmental adaptive capability, mental health problems may come to them. Some students also reflect that it is more difficult to study at home because it requires higher self-discipline. When students feel they are unable to make themselves study efficiently, the problem of self-negation and depression will increase. The last challenge is the uncertainty of examinations and school arrangements while studying online. For example, the Internet condition may have a 
problem during the exam. These uncertainties distract students' attention, decline their efficiency and make it hard to focus on studying compared with the environment on campus. As the result, students' academic grades may decrease and their mental health problem might increase.

The meaning maintenance model (MMM) provided a concept for people to understand the relationship between COVID-19 and mental health problems among Chinese college students. People have a need to certify meaning, which means people receive information and build their perceptions based on the information of the external world. When unexpected changes occurred in the external world, their formal perception will also be changed [3]. Hence, people have to form a new internal world based on the changes. When this happened, people may feel aversion, with a high probability leading to a psychological problem [4]. In the present research paper, the unexpected situations are COVID-19, lockdown, and study online. COVID-19 changed people's lifestyle and the relationship with family, friends, and neighborhoods, and it forced students to accept the new situation and to rebuild an internal world to modify their mental health.

In addition, college students are in the transition between adolescence and adulthood. Students at this stage are immature, so they are easy to be affected by the environment. One of the well-known theories is called Bronfenbrenner's ecosystem theory, which explains the different impacts of social development on one's development. The theory clarified that the environment you are in will affect who you are. Social factors could play a decisive role in the way you think, the emotion you feel, and the type of preferences. People changed when the environment is changed. There are five systems included in the theory: microsystems, mesosystem, exosystem, macrosystem, and chronosystem. Each system refers to a different environment in society. Microsystem indicates the group of people that contract directly. For example, parents, friends, and teachers. Mesosystem is the connection between the microsystem and exosystem. Exosystem shows the indirect factor that affects people. For example, mass media, and social welfare services. Macrosystem determines the cultural elements that affect life. Chronosystem refers to people's current developmental stage and the situation they are experiencing. Therefore, even tiny changes within each system could bring variance in different systems and interact between each other, and then cause serious psychological problems.

From the perspective of Bronfenbrenner's ecosystem theory, chronosystem can be referred to as COVID19 which is the current situation where students are experiencing. Parents are the microsystem for students since they are the direct people they communicate with. Each media and news are an exosystem. Students read and hear tons of news about COVID-19 every day, and doing so will change their own view of COVID-19. Finally, Chinese people like to gather around and celebrate festivals, however, during the pandemic and lockdown, people changed their way of celebrating. This can refer to macrosystem.

Everyone has been threatened by the virus, and the general atmosphere was intense. The original world had been changed. Peers, family, workplace, or mass media were not simply influenced, but they interacted with each other under these special and unhealthy life events, creating the general environment for individuals, where one will finally affected who they were, including affecting their mental status. Considering these factors, the present study hypothesized that there was an increase in mental health problems among Chinese college students during COVID-19. By reviewing existing research articles, the present study aimed to find some related evidence. After reviewing, the finding to examine whether our hypothesis is true or not would be summarized.

\section{MENTAL HEALTH PROBLEM AND IN- TERVENTION APPROACHES DURING COVID-19}

\subsection{Mental health problem}

In this part, several research articles supporting the hypotheses have been found. The articles chose to support the hypotheses are from three aspects, which include: sleeping problems, negative emotions, and PTSD of COVID-19 among Chinese college students.

To study whether there was an increase in depression during COVID-19 in Chinese college students, an online cross-sectional survey was conducted by [5]. They used the Center for Epidemiologic Studies Depression Scale, the Multi-Dimensional Scale of Perceived Social Support, the Herth Hope Index, and the self-designed issues to create an electronic questionnaire. In the total of 1681 participants, the rate of depressive symptoms was $56.8 \%$ (955) which was higher than the general population. Considering the result, college students who are in grades third, fourth, and fifth years showed a significantly higher rate of depressive symptoms $(P=0.010)$ compared with those in grades one, and second years. The survey indicates COVID-19 did have a high prevalence of depressive symptoms among Chinese college students.

Another study conducted by [6] studied negative emotions during COVID-19 among Chinese college students. The research used the Depression Anxiety Stress Scale, with 21-self-reported items(DASS-21). The scale included three parts: stress, anxiety, and depression. The rate in each part was from 0 (totally disagree) to 3 (totally agree). The higher the score of stress, 
anxiety, and depression, the serious the negative emotions. As the result, nearly $24.9 \%$ of college students have anxiety symptoms.

The other study evaluated insomnia among Chinese college students during COVID-19. The researchers conducted an online survey with a total of 11,835 participants. The survey used the Pittsburgh Sleep Quality Index (PSQI) which is a self-rated questionnaire that has seven aspects of sleep quality during a period. For example, subjective sleep quality, sleep latency, and sleep duration [7]. The total score of PSQI was ranging from 0 to 21 . Additionally, there were 19 items rated on a scale scored from 0 (not during the past month) to 3 (>=3 times a week). The result of the survey was $23.2 \%$ which is a high prevalence of sleeping problems during COVID-19. The study indicated that college students were more likely to have sleeping problems, such as sleep disturbances and later bedtimes. Students with insomnia should use sleep medication to regulate sleep.

[8] studied the prevalence of PTSD among college students in China during COVID-19. The study investigated the result by using online survey versions of the PTSD Checklist Civilian Version(PCL-C) and the 9question Patient Health Questionnaires(PHQ-9). In the first four weeks, the PTSD was measured using 17 items on a five-point Likert scale ranging from 1 (not at all) to 5 (extremely) with a total score from 17 to 85 . A higher score determined a higher level of PTSD, and a score of 38 or higher will be considered probable PTSD. The prevalence of PTSD was $2.7 \%$ in a total of 2485 participants. This study can be related to the last research studied by [7]. It showed PTSD was strongly associated with the sleeping problem during COVID-19. Moreover, the study pointed out the fear of COVID-19 and the anxiety of being graduated are the main factors of having PTSD symptoms.

According to these articles, the hypothesis of there is an increase during COID-19 among Chinese college students is tenable. Each article used experiments and collected authentic data among a certain amount of people to verify the mental health problem during COVID19. And the overall conclusion from these reviewed literature is that COVID-19 did caused significant increase of college students' mental health problems.

\subsection{Prevention of Mental Health}

Since COVID-19 influenced people not only physically, but also mentally. Plenty of articles have determined ways to help Chinese college students to improve mental health. Below are the articles about how to improve mental health problems during COVID-19.

Since plenty of people are under enormous psychological pressure which could lead to anxiety, depression, insomnia and other mental health problems [9]. The study determined professional psychology teams can help people decrease their negative emotions. During the pandemic, the National Health Commission of China has organized a call for emergency psychological crisis intervention. Hence, various expert teams formed from mental health associations and organizations to redact guidelines and public articles/videos about mental health and online mental health services to help people. In this case, college students can call up or speak to psychologists online to remit their pressure and prevent mental health problems.

The other study showed physical activities have a strong relationship with psychological problems. The level of physical activity was measured using the short form of the International Physical Activity Questionnaire(IPAQ-SF). The questionnaire included ten items on specific home activities, such as running, yoga, household chores and so on. Participants reported their physical activity per week to help researchers collect data. The questionnaire found approximately $52.3 \%$ of Chinese college students had a shortage of physical activity. The result also showed participants with a high level $(\beta=-0.121, P<0.001)$ of physical activity had low anxiety. Moreover, the study pointed out that college students need to have appropriate physical activities during COVID-19 to protect their psychological health [10].

Another study listed four pieces of advice that can help college students cope with mental health problems. The first one was to reduce the time of receiving news from the official and unofficial channels. Seeing too much news about COVID-19 would make students feel anxious. The second piece of advice was to increase communication with friends, family members, and loved ones. Although students were quarantined at home, try to use social media to connect with them. Communicating with close people will help students reduce loneliness and some psychological problems. The third piece of advice was to maintain your daily routine. Students can try to do the "healthy" routine. For instance, go to bed on time and have three meals each day. The fourth one was to think about the benefit of being isolated. If students are under negative emotions, they can try to think optimistically. Instead of thinking about the disadvantages of isolation, students can think advantages of isolation. For example, quarantine help to protect people's health [11].

This part concludes several ways to improve mental health problems during COVID-19. Such as talking with professionals, doing physical activities, and maintaining a healthy daily routine. These ideas aimed to help both people who have mental health problems and those who do not to regulate their psychological health. 


\section{CONCLUSION}

During the time of COVID-19, the number of college students' mental health problems increased strikingly. The ideas of discovering mental health problems and intervening in these problems are important for not only professionals, but also the school, family, and society. Regular mental services should be provided in schools and communities, furthermore, the importance of mental health should be publicized more by the government and other authorities to increase the popularizing rate between citizens. More settings of mental services are not only for COVID-19 but also for a future pandemic. Additionally, the school needs to teach more knowledge about mental health to let students acquaint mental health problems and have a certain ability to regulate these problems once they are facing these challenges.

All these studies have investigated the impact of COVID-19 on Chinese college students scientifically. However, there are still limitations. For example, the sample of students is not diverse enough. More of the participants have come from Han nationality. Only a few participants are from other nationalities. Therefore, the research could not study the examples and collect data from a well-generalized population. Moreover, all the questionnaires were finished online. Participants may not take the survey seriously, and there are uncertainties about the answers since participants have a chance to lie about it.

\section{REFERENCES}

[1] World Health Organization. (n.d.). Coronavirus disease (COVID-19). World Health Organization. Retrieved September 26, 2021, from https://www.who.int/news-room/q-adetail/coronavirus-disease-covid-19.

[2] Chen, R.-ning, Liang, S.-wei, Peng, Y., Li, X.-guo, Chen, J.-bin, Tang, S.-yao, \& Zhao, bo. (2020, August 15). Mental health status and change in living Rhythms among college students in China during the Covid-19 pandemic: A large-scale survey. Journal of Psychosomatic Research. Retrieved September 26, 2021, from https://www.sciencedirect.com/science/article/pii/S 0022399920307819?via\%3Dihub.

[3] KD; H. S. J. P. T. V. (2006). The meaning maintenance model: On the coherence of social motivations2006. Personality and social psychology review : an official journal of the Society for Personality and Social Psychology, Inc. Retrieved September 26, 2021, from https://pubmed.ncbi.nlm.nih.gov/16768649/.
[4] Zuo, S., HUANG, N., WANG, F., \& CAI, P. (n.d.). Meaning maintenance model: The development of theory and research challenges. China science journal. Retrieved September 26, 2021, from http://pub.chinasciencejournal.com/AdvancesinPsy chologicalScience/43004.jhtml.

[5] Yu, M., Tian, F., Cui, Q., \& Wu, H. (2021, January 29). Prevalence and its associated factors of depressive symptoms among Chinese college students during the COVID-19 pandemic. BMC Psychiatry. Retrieved September 26, 2021, from https://link.springer.com/article/10.1186/s12888021-03066-9.

[6] Zhang, Y., Zhang, H., Ma, X., \& Di, Q. (2020, May 25). Mental health problems during the COVID-19 pandemics and the mitigation effects of exercise: A longitudinal study of college students in China. MDPI. Retrieved October 1, 2021, from https://www.mdpi.com/1660-4601/17/10/3722.

[7] Zhou, S.-J., Wang, L.-L., Yang, R., Yang, X.-J., Zhang, L.-G., Guo, Z.-C., Chen, J.-C., Wang, J.-Q., \& Chen, J.-X. (2020, June 6). Sleep problems among Chinese adolescents and young adults during the coronavirus-2019 pandemic. Sleep Medicine. Retrieved October 3, 2021, from https://www.sciencedirect.com/science/article/pii/S 1389945720302550.

[8] Tang, W., Hu, T., Hu, B., Jin, C., Wang, G., Xie, C., Chen, S., \& Xu, J. (2020, May 13). Prevalence and correlates of PTSD and depressive symptoms one month after the outbreak of the covid-19 epidemic in a sample of home-quarantined Chinese University students. Journal of Affective Disorders. Retrieved October 3, 2021, from https://www.sciencedirect.com/science/article/pii/S $016503272030879 X$.

[9] Li, W., Yang, Y., Liu, Z.-H., Zhao, Y.-J., Zhang, Q., Zhang, L., Cheung, T., \& Xiang, Y.-T. (2020, March 15). Progression of mental health services during the COVID-19 outbreak in China. International journal of biological sciences. Retrieved October $\quad 4, \quad 2021, \quad$ from https://www.ncbi.nlm.nih.gov/pmc/articles/PMC70 98037/.

[10] Xiang, M.-Q., Tan, X.-M., Sun, J., Yang, H.-Y., Zhao, X.-P., Liu, L., Hou, X.-H., \& Hu, M. (1AD, January 1). Relationship of physical activity with anxiety and depression symptoms in chinese college students during the covid-19 outbreak. Frontiers. Retrieved September 26, 2021, from https://www.frontiersin.org/articles/10.3389/fpsyg. 2020.582436/full?utm_source=researcher_app\&ut 
$m \_$medium $=$referral $\& u t m \_$campaign $=R E S R \_M R K$

T_Researcher_inbound.

[11] Fiorillo, A., \& Gorwood, P. (2020, April 1). The consequences of the COVID-19 pandemic on Mental Health and implications for clinical practice: European psychiatry. Cambridge Core. Retrieved October 4, 2021, from https://www.cambridge.org/core/journals/european -psychiatry/article/consequences-of-the-COVID19pandemic-on-mental-health-and-implications-forclinical-

practice/E2826D643255F9D51896673F205ABF28. 